\section{Evaluation of irisin, retinol-binding protein 4, and leptin serum levels as biomarkers of macrovascular complications involvement in Saudi type 2 diabetes mellitus. A case-control study}

\author{
Ehdaa Y. Ali, MSc, Gehan A. Hegazy, MSc, MD, \\ Enayat M. Hashem, $M S c, M D$.
}

\section{ABSTRACT}

Objectives: To evaluate adipokine serum values of irisin, retinol-binding protein 4, and leptin in Saudi cases with type 2 diabetes mellitus (T2DM) for providing markers of T2DM macrovascular complications.

Methods: This case-control research was carried out at Erfan Hospital, Jeddah, Saudi Arabia. The study included 138 subjects, classified into 3 groups: 46 T2DM patients with macrovascular complications, 46 T2DM without macrovascular complications, and 46 controls. Participants evaluated clinically and some biochemical parameters were measured.

Results: Diabetic with and without macrovascular complications showed elevation of retinol-binding protein 4 (RBP4) and leptin; decreased irisin serum levels versus controls. Serum irisin was lower $(p=0.007)$, while RBP4 was higher $(p<0.0001)$ in T2DM patients with macrovascular complications versus without. Irisin showed negative correlations with fasting blood glucose (FBG), insulin, homeostatic model assessment of insulin resistance (HOMA-IR), RBP4, hemoglobin A1C (HbA1C), triglyceride, cholesterol, and low-density lipoprotein cholesterol. While RBP4 showed positive correlations with fasting blood glucose, insulin, HOMA-IR, leptin, and HbA1c; but a negative association with high-density lipoprotein cholesterol.

Conclusion: Type 2 DM patients had raised RBP4 and leptin, but lower irisin levels versus controls. Irisin was lower, but RBP4 was higher in T2DM patients with macrovascular complications versus without, suggesting T2DM patients in pro-inflammatory conditions. These results suggested that irisin is protective, while RBP4 is a risk factor for T2DM macrovascular complications.

Keywords: irisin, leptin, retinol-binding protein 4, macrovascular complications, type 2 diabetes mellitus

Saudi Med J 2020; Vol. 41 (12): 1369-1374 doi: 10.15537/smj.2020.12.25461
$\mathrm{D}$ abetes mellitus (DM) is a metabolic defect caused by impaired insulin action, secretion, or both. Approximately 451 million people globally diabetic, it is predicted that by 2040 approximately 693 million people (9.9\%) will have type 2 diabetes mellitus (T2DM) constituents $85-90 \%$ of diabetes cases. ${ }^{1}$ Kingdom of Saudi Arabia (KSA) has the second-highest rate of T2DM prevalence in the Middle East. ${ }^{2}$ The inflammatory process is one of the T2DM pathophysiological mechanisms. Throughout glycemic control deterioration, various factors affect the endothelium leading to endothelial dysfunction that deteriorates with insulin resistance (IR); these conditions are associated with T2DM macrovascular complications (MVC). Cardiovascular diseases (CVD) risk increased 2-fold to 4-fold in T2DM patients. ${ }^{3}$

Adipose tissue acts as an endocrine organ through inflammatory adipokines secretion that participates in IR in various tissues. Irisin is myokine that is proteolytically separated from transmembrane fibronectin type III domain-containing protein 5 (FNDC5). It has beneficial actions on metabolism. Irisin is inversely associated with inflammation that leads to IR. ${ }^{4}$ Adipocytokine retinol-binding protein 4 (RBP4) is a $21-\mathrm{kDa}$ protein of the lipocalin superfamily. It is only circulatory retinol (vitamin A) carrier protein and encoded by the RBP4 gene, localized in 10q23-q24 chromosome. ${ }^{5}$ Researchers reported that RBP4 was related to obesity-associated with metabolic defects, and serum RBP4 positively associated with subclinical carotid intima-media thickness and atherosclerosis in T2DM. ${ }^{6}$ Leptin is an adipokine hormone encoded by the ob gene. It has a role in adiposity and thermogenesis. Leptin and its receptor isoforms are expressed in adipose tissue and cardiovascular tissues as cells of smooth muscles, endothelial cells, and cardiomyocytes. Hyperleptinemia is associated with obesity, T2DM, and IR. Leptin peripheral effects enhanced inflammatory reactions, atherogenesis, oxidative stress, and thrombosis, leading to arterial wall stiffness, endothelial defects, and atherosclerotic plaques development. ${ }^{7}$

This study aimed to search for adipokines levels as irisin, RBP4, and leptin in T2DM patients and their association with MVC to provide reasonable MVC new markers in T2DM.

Methods. This case-control study was conducted at Erfan Hospital, Jeddah, Saudi Arabia, between January 2018-2019. The research was confirmed by the Biomedical Ethics Research Committee at King Abdulaziz University (Approval \#62-15) that was formed according to the Declaration of Helsinki. 
All patients were randomly chosen from outpatient endocrinology and cardiology clinics at Erfan Hospital. All participants signed informed consent to participate in the research. Excluded from the research were patients with T1DM, inflammatory diseases as bronchial asthma, gout, rheumatoid arthritis, ulcerative colitis, hepatitis, thyroiditis, nephritis, multiple sclerosis, allergic reactions, Crohn's disease, lupus erythematosus, congenital CVD or CVD caused by trauma, CVD diagnosed before T2DM, or cancer treated by chemotherapy or chronic kidney diseases.

The sample size was estimated before the study using the Raosoft software package for sample size calculation. By assuming that $50 \%$ of patients will have the disease, the required sample size was 92 patients at a 5\% error margin and a $95 \%$ confidence interval (CI).

A total of 138 subjects of both genders between 40 and 80 years old were included. Subjects were categorized into $46 \mathrm{~T} 2 \mathrm{DM}$ patients without MVC, 46 T2DM patients with MVC, and 46 healthy subjects that matched patients in age, gender, and body mass index (BMI) served as control. Type 2 DM diagnosis was based upon the American Diabetic Association's (ADA) criteria of fasting blood glucose level $\geq 7.0 \mathrm{mmol} / \mathrm{L}$. Macrovascular complications was defined based on the patient's medical profile and diagnosed based upon American Heart Association criteria (AHA). Macrovascular complications included coronary artery diseases (CAD) established by coronary angiography, peripheral vascular diseases (PVD) diagnosed by Doppler ultrasound of lower limb vessels, MVC diagnosed by brain-computed tomography scan, and carotid doppler examination. Cases with a history of myocardial infarction, coronary artery bypass, peripheral arteries diseases, apoplexy incidents, electrocardiogram abnormalities that suggested angina pectoris or ischemia, or angiographically established CAD were recruited to the MVC group.

All participants submitted full medical history and subjected to thorough clinical evaluation. Body weight $(\mathrm{kg})$ and height $(\mathrm{cm})$ of all subjects were taken and BMI was estimated. Blood pressure $(\mathrm{mmHg})$ was estimated. After fasting for 12 hours samples were gathered from all patients into ethylenediaminetetraacetic acid tubes for plasma collection needed for routine biochemical

Disclosure. Authors have no conflict of interests, and the work was not supported or funded by any drug company. tests and plain tubes for serum separation. Routine biochemical tests as glycated hemoglobin (HbA1C), fasting blood glucose (FBG), fasting insulin, lipid profiles as triglycerides, cholesterol, high-density lipoprotein-cholesterol (HDL-C), and serum creatinine were performed by the automatic biochemical analyzer (Olympus, AU 400, Tokyo, Japan). High-density lipoprotein-cholesterol, TC, and TG levels were utilized to calculate LDL-C. Insulin resistance (HOMA-IR) was calculated. Enzyme-linked immunosorbent assay (ELISA) kits from BioVendor GmbH in Heidelberg, Germany were utilized for serum levels evaluation of irisin (Cat\# RAG005R), RBP4 (Cat\# RAG018R), and leptin (Cat\# RD191991199).

Statistical analysis. Data were presented as mean \pm standard deviation (SD) and manipulated with IBM SPSS Statistics for Windows, version 23 (IBM SPSS, IBM Corp., Armonk, NY, USA). Shapiro-Wilk test was utilized to evaluate normal data distribution. Comparisons between groups were made using one-way analysis of variance (ANOVA) then by least-significant difference test (LSD) for normally distributed variables and Mann-Whitney U-test for abnormally distributed variables. Correlations between different measured parameters made using Pearson's correlations. Binary logistic regression analysis was utilized to find risk factors for macrovascular complications in T2DM. Data were statistically significant if $p$-values $<0.05$.

Results. Fasting blood glucose, fasting insulin, HOMA-IR, HbA1C, RBP4, and leptin values in T2DM cases without and with MVC complications were significantly elevated versus controls $(p<0.0001)$. Cholesterol values were elevated in T2DM cases without MVC versus controls and T2DM with MVC $(p=0.002$ and $p=0.022$ ). Retinol-binding protein 4 values were significantly decreased in T2DM cases with MVC versus those without MVC $(p<0.0001)$ HDL-C and irisin values in T2DM patients without and with MVC complications were significantly declined versus controls. Irisin levels were significantly declined in T2DM with MVC complications versus those without $\operatorname{MVC}(p=0.007)$ (Table 1).

In all participants, there were negative associations between irisin levels and RBP4, FBG, insulin, HOMA-IR, HbA1C, cholesterol, triglyceride, LDL-C. Serum RBP4 showed positive correlations with FBG, insulin, HOMA-IR, and $\mathrm{HbA1C}$, but negative correlations with HDL-C. Serum leptin had positive correlations with BMI, FBG, insulin, HOMA-IR, and HbA1C (Table 2). 
Adipokines and type 2 diabetes mellitus ... Ali et al

Table 1 - Comparison of demographic and clinical characteristics and measured parameters in different studied groups.

\begin{tabular}{|c|c|c|c|}
\hline Variables & $\begin{array}{l}\text { Control } \\
(\mathrm{n}=46)\end{array}$ & T2DM without MVC $(n=46)$ & $\begin{array}{l}\text { T2DM with MVC } \\
(\mathrm{n}=46)\end{array}$ \\
\hline Age (years) & $52.33 \pm 8.78$ & $53.80 \pm 9.12$ & $55.89 \pm 9.58$ \\
\hline Significance & & ${ }^{*} p=0.441$ & ${ }^{*} p=0.064 ;{ }^{\dagger} p=0.277$ \\
\hline \multicolumn{4}{|l|}{ Gender } \\
\hline Male & $23(50.0 \%)$ & $23(50.0 \%)$ & $28(60.9 \%)$ \\
\hline Female & $23(50.0 \%)$ & $23(50.0 \%)$ & $18(39.1 \%)$ \\
\hline Significance & & & $p=0.395$ \\
\hline Body mass index $\left(\mathrm{kg} / \mathrm{m}^{2}\right)$ & $30.22 \pm 5.29$ & $28.97 \pm 5.98$ & $29.79 \pm 4.85$ \\
\hline Significance & & ${ }^{*} p=0.269$ & ${ }^{*} p=0.704 ;{ }^{\dagger} p=0.468$ \\
\hline Systolic blood pressure $(\mathrm{mmHg})$ & $124.63 \pm 9.80$ & $128.33 \pm 14.13$ & $123.70 \pm 11.37$ \\
\hline Significance & & ${ }^{*} p=0.139$ & ${ }^{*} p=0.707 ;{ }^{\dagger} p=0.064$ \\
\hline Diastolic blood pressure $(\mathrm{mmHg})$ & $74.72 \pm 7.36$ & $73.35 \pm 8.82$ & $72.93 \pm 8.08$ \\
\hline Significance & & ${ }^{*} p=0.419$ & ${ }^{*} p=0.294 ;{ }^{\dagger} p=0.807$ \\
\hline Fasting blood glucose (mg/dl) & $92.17 \pm 10.74$ & $175.04 \pm 41.22$ & $177.78 \pm 69.53$ \\
\hline Significance & & ${ }^{*} p<0.0001$ & ${ }^{*} p<0.0001 ;{ }^{\dagger} p=0.482$ \\
\hline Fasting insulin (mU/L) & $8.98 \pm 0.46$ & $25.3 \pm 4.05$ & $25.59 \pm 8.30$ \\
\hline Significance & & ${ }^{*} p<0.0001$ & ${ }^{*} p<0.0001 ;{ }^{\dagger} p=0.842$ \\
\hline$H O M A-I R$ & $1.98 \pm 0.28$ & $11.35 \pm 4.04$ & $12.46 \pm 8.76$ \\
\hline Significance & & ${ }^{*} p<0.0001$ & ${ }^{*} p<0.0001 ;{ }^{\dagger} p=0.522$ \\
\hline Serum creatinine & $0.7976 \pm 0.10166$ & $0.8352 \pm 0.15853$ & $0.8552 \pm 0.13698$ \\
\hline Significance & & $p=0.375$ & ${ }^{*} p=0.103 ; \dagger p=0.756$ \\
\hline$H b A_{I C}(\%)$ & $5.28 \pm 0.26$ & $7.95 \pm 1.72$ & $8.28 \pm 1.69$ \\
\hline Significance & & ${ }^{*} p<0.0001$ & ${ }^{*} p<0.0001 ; p=0.269$ \\
\hline Total Cholesterol (mg/dl) & $165.37 \pm 22.50$ & $190.70 \pm 45.13$ & $172.17 \pm 42.99$ \\
\hline Significance & & ${ }^{*} p=0.002$ & ${ }^{*} p=0.395 ;{ }^{\dagger} p=0.022$ \\
\hline Triglycerides (mg/dl) & $116.48 \pm 44.74$ & $135.78 \pm 54.55$ & $127.02 \pm 52.76$ \\
\hline Significance & & ${ }^{*} p=0.071$ & ${ }^{ } \mathrm{P}=0.322 ;{ }^{\dagger} \mathrm{P}=0.410$ \\
\hline High density lipoprotein cholesterol (mg/dl) & $56.15 \pm 12.93$ & $45.09 \pm 7.28$ & $42.41 \pm 11.02$ \\
\hline Significance & & ${ }^{*} p<0.0001$ & ${ }^{*} p<0.0001 ;{ }^{\dagger} p=0.269$ \\
\hline Low density lipoprotein cholesterol (mg/dl) & $113.65 \pm 16.65$ & $122.85 \pm 44.06$ & $122.71 \pm 38.28$ \\
\hline Significance & & ${ }^{*} p=0.210$ & ${ }^{*} p=0.217 ;{ }^{\dagger} p=0.986$ \\
\hline $\operatorname{Irisin}(\mu g / m l)$ & $0.65 \pm 0.13$ & $0.58 \pm 0.13$ & $0.50 \pm 0.14$ \\
\hline Significance & & ${ }^{*} p=0.021$ & ${ }^{*} p<0.0001 ;{ }^{\dagger} p=0.007$ \\
\hline Retinol binding protein-4 (pg/ml) & $99.60 \pm 35.18$ & $186.59 \pm 68.47$ & $262.42 \pm 58.80$ \\
\hline Significance & & ${ }^{*} p<0.0001$ & ${ }^{*} p<0.0001 ;{ }^{\dagger} p<0.0001$ \\
\hline Leptin $(n g / m l)$ & $21.67 \pm 5.41$ & $34.89 \pm 13.47$ & $34.43 \pm 10.52$ \\
\hline Significance & & ${ }^{*} p<0.0001$ & ${ }^{*} p<0.0001 ;{ }^{\dagger} p=0.828$ \\
\hline
\end{tabular}

Data were expressed as mean \pm standard deviation. A comparison was made using the one-way ANOVA test followed by the least significant difference test (LSD) of parametric data. The Chi-square test was used to compare gender frequency, $p<0.05$ was considered significant. T2DM: type 2 diabetes mellitus, MVC: macrovascular complications, ${ }^{*} p$ : comparison versus control; ${ }^{\dagger} p$ : comparison versus T2DM without CVD complications, $p$ : significance between groups. HOMA-IR: homeostatic model assessment of insulin resistance, HbA1c: hemoglobin A1c 
Macrovascular complications patients were $(\beta=0.014,95 \%$ CI $0.000-0.387, p=0.012)$. While evaluated with binary logistic regression analysis in which groups were taken as dependent, and adipokines, lipid profile, glycemic control as independent variables. Irisin was protective against MVC in T2DM patients RBP4 $(\beta=1.020,95 \%$ CI 1.011-1.1020, $p<0.0001)$ and cholesterol $(\beta=0.965,95 \%$ CI $0.981-1.000, p=0.049)$ were positive risk factors for MVC in T2DM patients (Table 3).

Table 2 - Correlations between different inflammatory markers and measured parameters in all participants.

\begin{tabular}{lcccccc}
\hline Variables & \multicolumn{2}{c}{ Irisin } & $\begin{array}{c}\text { Retinol binding } \\
\text { protein } 4\end{array}$ & Leptin \\
\hline Retinol binding protein 4 & -0.334 & $(0.0001)$ & & & \\
Leptin & -0.147 & $(0.085)$ & 0.426 & $(0.0001)$ & & \\
Body mass index & -0.027 & $(0.756)$ & -0.019 & $(0.825)$ & 0.220 & $(0.009)$ \\
Fasting blood glucose & -0.209 & $(0.014)$ & 0.489 & $(0.0001)$ & 0.318 & $(0.0001)$ \\
Fasting insulin & -0.251 & $(0.003)$ & 0.629 & $(0.0001)$ & 0.396 & $(0.015)$ \\
HOMA-IR & -0.209 & $(0.014)$ & 0.531 & $(0.0001)$ & 0.316 & $(0.015)$ \\
HbA & -0.252 & $(0.003)$ & 0.479 & $(0.0001)$ & 0.375 & $(0.0001)$ \\
Total cholesterol & -0.220 & $(0.009)$ & 0.008 & $(0.921)$ & 0.157 & $(0.066)$ \\
Triglyceride & -0.246 & $(0.004)$ & 0.020 & $(0.815)$ & 0.140 & $(0.102)$ \\
High density lipoprotein cholesterol & 0.146 & $(0.087)$ & -0.409 & $(0.0001)$ & -0.158 & $(0.064)$ \\
Low density lipoprotein cholesterol & -0.244 & $(0.004)$ & 0.029 & $(0.740)$ & 0.068 & $(0.427)$ \\
\hline $\begin{array}{l}\text { Data were expressed as the correlation coefficient } \\
\text { the Person correlation coefficient. } p<0.05 \text { was considered significant. HOMA-IR: homeostatic model } \\
\text { assessment of insulin resistance }\end{array}$ & & & & \\
\hline
\end{tabular}

Table 3 - Binary regression analysis of risk factors in type 2 diabetes mellitus patients with macrovascular complications.

\begin{tabular}{lccc}
\hline Independent predictors & $\beta$ & $P$-values & $95 \%$ CI \\
\hline Irisin & 0.014 & 0.012 & $0.000-0.387$ \\
Retinol binding protein 4 & 1.020 & 0.0001 & $1.011-1.029$ \\
Leptin & 0.997 & 0.851 & $0.963-1.031$ \\
Body mass index & 1.029 & 0.468 & $0.953-1.110$ \\
Fasting blood glucose & 1.001 & 0.816 & $0.994-1.008$ \\
Fasting insulin & 1.006 & 0.846 & $0.945-1.072$ \\
HOMA-IR & 0.435 & 1.025 & $0.964-1.090$ \\
HbA & 1.121 & 0.360 & $0.978-1.430$ \\
Total cholesterol & 0.990 & 0.049 & $0.981-1.000$ \\
Triglyceride & 0.997 & 0.431 & $0.989-1.005$ \\
High density lipoprotein cholesterol & 0.969 & 0.173 & $0.927-1.014$ \\
Low density lipoprotein cholesterol & 1.000 & 0.988 & $0.990-1.010$ \\
\hline
\end{tabular}

Data were expressed as the correlation coefficient $(r)$ and significance. Correlations were made using the Person correlation coefficient. $p<0.05$ was considered significant. HOMA-IR: homeostatic model assessment of insulin resistance, HbA1c: hemoglobin A1c 
Discussion. Considering the pro-inflammatory state of diabetes, several adipokine inflammationrelated factors as irisin, RBP4, and leptin in T2DM cases with and without MVC were analyzed. This study revealed a decrease in serum irisin in T2DM subjects with and without MVC that confirmed irisin's potential role in glucose metabolism regulation. Serum irisin was significantly declined in T2DM with MVC versus without MVC. Type $2 \mathrm{DM}$ cases with MVC have more inflammation in their vascular tissues than those without MVC. Zhang et $\mathrm{al}^{8}$ reported a significant decline in serum irisin in T2DM and showed a further decrease in serum irisin when MVC existed. Anastasilakis et $\mathrm{al}^{4}$ reported lower serum irisin in MI and CAD cases than controls. Changes in activity and expression of peroxisome proliferator-activated receptor-gamma coactivator 1 -alpha (PGC-1 $\alpha$ ) may explain irisin level reduction in T2DM cases. Irisin is stimulated by PGC- $1 \alpha$ expression. Meanwhile, in T2DM, PGC- $1 \alpha$ expression was down-regulated in skeletal muscles. Therefore, reduced serum irisin values in T2DM patients are caused by reduced PGC- $1 \alpha$ synthesis and function in their muscles. ${ }^{9}$ Furthermore, the experimental animal model suggested that during ischemic cardiomyopathy, decreased FNDC5 expression in skeletal muscle is due to inflammatory cytokines. ${ }^{10}$ Low levels of irisin in T2DM with MVC cases in this research could be explained by that irisin energy hemostasis controlling effects as most secreted irisin used to control disturbed metabolism. In addition, in this study, significant negative associations between serum irisin and $\mathrm{HbA} 1 \mathrm{C}$, cholesterol, triglyceride, and LDL-C were found. Moreover, raised irisin values were related to a decreased risk of MVC in T2DM $(\beta=0.014$, 95\%CI $\quad 0.000-0.387, \quad p=0.012)$. Retinol-binding protein 4 is the chief circulatory transporter protein for retinol (vitamin A). Retinol-binding protein 4 was found to be genetically linked to T2DM risk. In this research, serum RBP4 values were significantly higher in T2DM cases with and without MVC than in controls. A case-control study showed that serum RBP4 values were more elevated in T2DM cases in comparison to the controls and RBP4 strongly related to LDL, therefore it is considered as a risk factor for CVS development. ${ }^{6}$ Kadoglou et $\mathrm{al}^{11}$ found that serum RBP4 values were higher in established carotid atherosclerosis cases than controls. Our data showed that serum RBP4 levels were higher in T2DM cases with MVC than T2DM cases without MVC. Zhang et $\mathrm{al}^{8}$ reported that RBP4 values were significantly increased in T2DM cases with MVC versus those without MVC. Declined expression of glucose transporter 4 in adipose tissue in a diabetic patient was related to increased RBP4 expression in adipose tissue and raised RBP4 serum values. Increased circulating RBP4 raised insulin resistance by suppressing insulin signaling in muscle and raising liver glucose output. ${ }^{8}$ The results of this study showed that RBP 4 had negative associations with irisin and HDL-C but had positive associations with FBG and HbA1C. Moreover, increased RBP4 values were associated with increased MVC risk in T2DM $(\beta=1.020,95 \%$ CI $1.011-1.029$, $p<0.0001)$. Kwanbunjan1et al. found strong positive correlations between RBP4 and TG in T2DM cases moreover, they found a negative association between HDL-C and RBP4. ${ }^{12}$

Multiple fields of research have studied the correlation between leptin and diabetes mellitus and the results have been variant. High leptin values were reported related to adiposity and different CVD risk factors. Additionally, they are associated with IR and increased T2DM risk. In this study, the results revealed that serum leptin was higher in T2DM cases with and without MVC than in controls. Also, similar results were reported by Al Sheikh, who studied serum leptin levels in correlation with T2DM in a sample of Saudi males; she found a significant rise in serum leptin in the diabetic group versus non-diabetic group. ${ }^{7}$ In accordance with our results, the study of Memon et $\mathrm{al}^{13}$ studied serum values of adipocytokines and advanced glycation end products in diabetic and non-diabetic cases with myocardial infarction, and they reported that T2DM patients with or without MI had significantly higher leptin values than controls. ${ }^{13}$ In this study, leptin had positive associations with RBP4, BMI, FBG, and HbA1C. Similar results have been achieved by Memon et al, ${ }^{13}$ reported that there was a significant positive correlation between leptin and BMI and FBG in T2DM patients they explained their results by that inflammation is playing a pivotal role in cardiovascular complication the pathogenesis in T2DM.

Study limitations. This study has lack of registration of waist-hip circumference as an indicator of visceral obesity. Lastly, lack of estimation of highly sensitive CRP that is potential markers of cardiovascular diseases.

In conclusion, possible beneficial anti-inflammatory properties of irisin and the harmful effects of RBP4 in T2DM patients with MVC. Association between irisin with markers of glucose homeostasis and lipid profile suggested that they may be a predictor of and protective factor against developing DM. More studies are needed with a larger number of patients to prove possible diagnostic, prognostic, and therapeutic roles for irisin 
and RBP4 in T2DM with MVC and explain possible mechanisms that contribute to 3 studied markers levels in T2DM.

\section{Received 28th July 2020. Accepted 29th September 2020.}

From the Biochemistry Department (Ali), Faculty of Science, University of Jeddah, from the Clinical Biochemistry Department (Hegazy), Faculty of Medicine, King Abdulaziz University, Jeddah, Kingdom of Saudi Arabia; from the Medical Biochemistry Department (Hegazy), National Research Center, Cairo, and from the Medical Biochemistry and Molecular Biology Department (Hashem), Faculty of Medicine, Alexandria University, Alexandria, Egypt.

Address correspondence and reprints request to: Dr. Gehan A. Hegazy, Department of Clinical Biochemistry, Faculty of Medicine, King Abdulaziz University Jeddah, Kingdom of Saudi Arabia. E-mail: gehanhegazy@hotmail.com ORCID ID: https://orcid.org/0000-0001-7918-6423

\section{References}

1. Lovic D, Piperidou A, Zografou I, Grassos H, Pittaras A, Manolis A. The growing epidemic of diabetes mellitus. Curr Vasc Pharmacol 2020; 18: 104-109.

2. Hegazy GA, Awan Z, Hashem E, Al-Ama N, Abunaji AB. Levels of soluble cell adhesion molecules in type 2 diabetes mellitus patients with macrovascular complications. J Int Med Res 2019: 0; 1-11.

3. Price AH, Weir CJ, Welsh P, McLachlan S, Strachan MWJ, Sattar N, et al. Comparison of non-traditional biomarkers, and combinations of biomarkers, for vascular risk prediction in people with type 2 diabetes: The Edinburgh type 2 diabetes study. Atherosclerosis 2017; 264: 67-73.

4. Anastasilakis AD, Koulaxis D, Kefala N, Polyzos SA, Upadhyay J, Pagkalidou E, et al. Circulating irisin levels are lower in patients with either stable coronary artery disease (CAD) or myocardial infarction (MI) versus healthy controls, whereas follistatin and activin A levels are higher and can discriminate MI from CAD with similar to CK-MB accuracy. Metabolism 2017; 73: 1-8.
5. Lamiquiz-Moneo I, Bea AM, Palacios-Pérez C, De MiguelEtayo P, González-Gil EM, López-Ariño C, et al. Effect of lifestyle intervention in the concentration of adipoquines and branched chain amino acids in subjects with high risk of developing type 2 diabetes: Feel4Diabetes Study. Cells 2020; 9: 693.

6. Wessel H, Saeed A, Heegsma J, Connelly MA, Faber KN, Dullaart RP. Plasma levels of retinol binding protein 4 relate to large VLDL and small LDL particles in subjects with and without type 2 diabetes. J Clin Med 2019; 8: 1792.

7. Al Sheikh MH. The determinants of leptin levels in diabetic and nondiabetic Saudi Males. Int J Endocrinol 2017; 2017: 3506871 .

8. Zhang M, Chen P, Chen S, Sun Q, Zeng QC, Chen JY, et al. The association of new inflammatory markers with type 2 diabetes mellitus and macrovascular complications: a preliminary study. Eur Rev Med Pharmacol Sci 2014; 18: 1567-1572.

9. Stanford KI, Goodyear LJ. Muscle-adipose tissue cross talk. Cold Spring Harb Perspect Med 2018; 8: a029801.

10. Matsuo Y, Gleitsmann K, Mangner N, et al. Fibronectin type III domain containing 5 expression in skeletal muscle in chronic heart failure-relevance of inflammatory cytokines. J Cachexia Sarcopenia Muscle 2015; 6: 62-72.

11. Kadoglou NP, Lambadiari V, Gastounioti A, Gkekas C, Giannakopoulos TG, Koulia K, et al. The relationship of novel adipokines, RBP4 and omentin-1, with carotid atherosclerosis severity and vulnerability. Atherosclerosis 2014; 235: 606-612.

12. Kwanbunjan K, Panprathip P, Phosat C, et al. Association of retinol binding protein 4 and transthyretin with triglyceride levels and insulin resistance in rural thais with high type 2 diabetes risk. BMC Endocr Disord 2018; 18: 26.

13. Memon AG, Rahman MA, Alghasham A, Hamid S, Hamid Q. Correlation between adipocytokines and AGE products in diabetic and non-diabetic patients with myocardial infarction. Res Chem Intermed 2015; 41: 1625-1634. 